\title{
Flow analysis in right-angled pipes with different geometric shapes and parameters for sludge discharge in slime shield machines measured using a CFD-DEM method
}

\author{
Lumin Chen, Kaixuan Liu, Yufeng Yao, Jun Yao, Zixue Su, Guofu Luo
}

\begin{abstract}
Mud and gravel deposition often occurs in circulation systems of pumps. In this paper, a liquid-solid coupling method combining CFD and DEM is proposed to study two-phase flow behavior of water, The flow characteristics of water-clay mud and the motion characteristics of gravel were simulated under different discharge pipe bending radii and geometry shape conditions. Numerical results showed that for a circular cross section pipe with a fixed bending radius, the collision times between the gravel particles and the pipe wall decreased with the increase of the bending radius.
\end{abstract}

Keywords: Shield machine, muddy water circulation, solid-liquid coupling, discrete element method, computational fluid dynamics

\section{Introduction}

In recent years, shield machines have been widely used in tunnel excavation engineering project, along with the acceleration of urban constructions particularly in China. Among those existing shield machines, the slurry shield excavator is often used in non-mountainous urban rail transit constructions because of its minimal disturbance to the ground and the soil near the excavation surface during the operation.

At present, significant advancements have been achieved in the research and development of shield tunneling machines by scientists and engineers around the world. For example, Huo et al. ${ }^{[1]}$ and $\mathrm{Li}$ et al. ${ }^{[2]}$ analyzed the mechanical properties of the cutterhead of the shield machine, and optimized the cutterhead to significantly reduce 
the equivalent stress and deformation of the cutterhead; Shi et al ${ }^{[3]}$ analyzed the torque of cutterhead of the EPB shield machine, and found that the cutter opening and earth pressure are two important factors affecting torque of the cutter; Qi et al ${ }^{[4]}$ studied the rock breaking process of the cutter of the road header, and determined the optimal knife spacing of the rock; Moeinossadat et al. ${ }^{[5]}, \mathrm{Zhu} 、 \mathrm{Li}^{[6]}$ and Li et al. ${ }^{[7]}$ studied the surface subsidence caused by shield tunneling, and proposed effective methods for predicting surface settlement; Ooishi et al. ${ }^{[8]}$ developed a shield bulldozer measuring device to facilitate accurate measurement of the discharged muck; Shi et al. ${ }^{[9-10]}$ and Yang et al. ${ }^{[11]}$ analyzed the energy conservation of the shield machine system to reduce the energy loss of the shield machine; Yang et al. ${ }^{[12-13]}$, Wang et al. ${ }^{[14-15]}$, Zhang et al. ${ }^{[16]}$ and Zhao et al. ${ }^{[17]}$ studied the dynamics of the shield thruster system which is helpful for the in-depth study of the dynamic characteristics of the shield thrust system;

Because the shield machine is large-scale equipment and its working environment is varying and also complicated during the underground excavation process. Qin and $\mathrm{Xia}^{[18]}$ calculated the pressure loss of the shield system of mud circulation, and determined the head of the slag pump; Zhai et al. ${ }^{[19]}$ studied the rheological properties of slurry used in slurry shields, and obtained a bentonite slurry with better comprehensive performance; Bie et al. ${ }^{[20]}$ studied the formation mechanism of slurry jam in shield machine and proposed a solution, and finally solved the problem of cutterhead and knife blockage; Dong ${ }^{[21]}$ studied the wear conditions and damping technologies of mud water pipelines, improved the wear resistance of pipelines and reduced the vibration of pipelines; Wang and Zhong ${ }^{[22]}$ analyzed the influence of slurry from the slag removal efficiency team of the slurry separation system, and proved that the mud screening efficiency is very important for the mud circulation system.; Zhou et al. ${ }^{[23]}$ conducted experimental research on backfill grouting for large borehole slurry shield tunnels, so that the discharged soil can be recycled as a grouting material for the sand layer, reducing construction costs.

Despite of many researches focused on the shield machine as mentioned above, to the authors' knowledge, there is very little working progress made on the mud circulation system in the slurry shield machines. The 
transport characteristics of the mud circulation system and its capability to carry muck are very important for the shield machine. If the circulation system has an undesirable ability, the muck in the shield machine will not be able to be carried and discharged in time. This can lead to the pipe being blocked, the machine being shut down and operation being stopped, etc. The consequence of financial impacts and losses will be at massive scale in most cases. Therefore, there is an urgent need to study the circulation system of the slurry shield machine and to improve the circulation system design performance of the slurry shield to reduce the probability of the clogging of the pipe.

In this paper, a generic slurry shield machine is used as an object to study its circulation system performance. Based on the overall analysis of the circulation systems of the shield machine, a section of the redirection ducts is chosen to evaluate the motion characteristics of the mud and the deposits in different shapes of the pipe bending. According to the obtained calculation results, the most favorable pipe bending shape for efficient discharging and slagging will be identified to provide the baseline reference for the sponsored company to further optimizate design of the shield machines.

\section{Mathematical models}

In this study, the discrete element software (EDEM) and the CFD slurry flow model supported by ANSYS Fluent are used to conduct the solid-fluid two-phase coupling simulation and analysis. The following introduce the theoretical models used for CFD simulation.

\subsection{Fluid motion equations}

Any fluid flow problem must satisfy the conservation of mass equation. That is if a closed area is taken in the flow field, the difference between the fluid mass flowing into and out of the surfaces of this so-called control volume $(C V)$ per unit time is equal to the change of the mass inside it. Its volumetric expression is: 


$$
\frac{\partial}{\partial t} \iiint_{V} \rho d x d y d z+\oiint_{A} \rho d A=0
$$

In equation (1), $V$ represents the control volume; $A$ represents the control surfaces. The differential form in the Cartesian coordinates is as follows:

$$
\frac{\partial \rho}{\partial t}+\frac{\partial(\rho u)}{\partial x}+\frac{\partial(\rho v)}{\partial y}+\frac{\partial(\rho w)}{\partial z}=0
$$

where $\rho$ is the density of the mud, $u, v, w$ are velocity components, $t$ is time, $x, y, z$ are Cartesian coordinates, respectively.

In the process of fluid motion, the fluid meets the conservation law of mass and at the same time it should satisfy the physical law of the conservation of momentum. Thus, muddy fluid continuity and momentum equations are:

$$
\begin{gathered}
\frac{\partial}{\partial t}\left(\bar{\alpha}_{c} \rho_{c}\right)+\frac{\partial}{\partial x_{j}}\left(\bar{\alpha}_{c} \rho_{c} \theta_{c j}\right)=0 \\
\frac{\partial}{\partial t}\left(\bar{\alpha}_{c} \rho_{c} \theta_{c i}\right)+\frac{\partial}{\partial x_{j}}\left(\bar{\alpha}_{c} \rho_{c} \theta_{c j} \theta_{c i}\right)=-\bar{\alpha}_{c} \frac{\partial \bar{p}}{\partial x_{i}} \\
+\frac{\partial \theta_{c i j}}{\partial x_{j}}+F_{d c i}+\bar{\alpha}_{c} \rho_{c} g-\frac{\partial}{\partial x_{j}}\left(\bar{\alpha}_{c} \rho_{c} v_{c i}^{\prime \prime} v_{c j}^{\prime \prime}\right)
\end{gathered}
$$

where $F_{d c i}=-\sum_{d} F_{d c, d i}$.

In the equations (3) and (4): $\bar{p}$ represents the total pressure of the fluid phase and the particle phase; $\rho_{c}$ represents the mass density of the fluid phase; $g$ denotes the acceleration of gravity; $\theta_{c i}$ and $\theta_{c i}$ indicate the turbulent viscosity coefficient of the fluid phase; $\bar{\alpha}_{c}$ represents the fluid phase volume fraction; $\varepsilon_{c i j}$ represents the fluid phase viscous stress; $\bar{\alpha}_{c} \rho_{c} v_{c i}^{\prime \prime} v_{c j}^{\prime \prime}$ represents the fluid phase Reynolds stress; $F_{d c, d i}$ represents the resistance of the fluid phase to the particle phase.

The continuity and momentum equations of the solid particle phase (e.g. gravel) are:

$$
\frac{\partial \bar{\alpha}_{d}}{\partial t}+\frac{\partial}{\partial x_{j}}\left(\bar{\alpha}_{d} \theta_{q i}\right)=0
$$




$$
\begin{aligned}
& \frac{\partial}{\partial t}\left(\bar{\alpha}_{d} \rho_{d} \theta_{q_{i}}\right)+\frac{\partial}{\partial x_{j}}\left(\bar{\alpha}_{d} \rho_{d} \theta_{q j} \theta_{q_{i}}\right)=-\bar{\alpha}_{d} \frac{\partial \bar{p}}{\partial x_{i}}+\frac{\partial \bar{p}_{d}}{\partial x_{i}} \\
& +\frac{\partial \varepsilon q_{d i j}}{\partial x_{j}}+F_{d c, d i}+F_{d, s i}+\bar{\alpha}_{d} \rho_{d} g_{i}+\frac{\partial}{\partial x_{j}}\left(\bar{\alpha}_{d} \rho_{d} v_{d i}^{\prime \prime} v_{d j}^{\prime \prime}\right)
\end{aligned}
$$

In the equations (5) and (6), $\bar{p}_{d}$ represents the solid pressure caused by collisions between the particles; $\rho_{d}$ represents the mass density of the particulate phase; $g_{i}$ represents the acceleration of the particle phase; $\theta_{q i}$ and $\theta_{q j} q_{j}$ represent the turbulent viscosity coefficient of the particle phase; $\bar{\alpha}_{d}$ represents the volume fraction of the particle phase; $q_{q j i}$ represents sand viscous stress caused by particle collisions and positional shifts; $\bar{\alpha}_{d} \rho_{d} v_{d i}^{\prime \prime} v_{d j}^{\prime \prime}$ represents the Reynolds stress of the particle phase; $F_{d, s i}$ represents the collision resistance between the particle phase $d$ and the particle phase $s ; \beta_{d c}$ represents the total pressure of the fluid and particulate phases; $\beta_{d s}$ represents the total pressure of the fluid and particulate phases;

The turbulence intensity is at moderate level for the fluid flow in the pipe. Using the standard two-equation $k-\varepsilon$ turbulence model, the governing equations for turbulence kinetic energy $(k)$ and turbulence dissipation rate $(\varepsilon)$ can be expressed as:

$$
\left\{\begin{array}{l}
\frac{\partial k}{\partial t}-\frac{\partial}{\partial x_{j}}\left(\frac{K_{m}}{\sigma_{k}} \frac{\partial k}{\partial x_{j}}\right)=K_{m}\left[\left(\frac{\partial u}{\partial x_{j}}\right)^{2}+\left(\frac{\partial v}{\partial x_{j}}\right)^{2}+\left(\frac{\partial w}{\partial x_{j}}\right)^{2}\right]-\varepsilon \\
\frac{\partial \varepsilon}{\partial t}-\frac{\partial}{\partial x_{j}}\left(\frac{K_{m}}{\sigma_{\varepsilon}} \frac{\partial \varepsilon}{\partial x_{j}}\right)=c_{1} K_{m}\left[\left(\frac{\partial u}{\partial x_{j}}\right)^{2}+\left(\frac{\partial v}{\partial x_{j}}\right)^{2}+\left(\frac{\partial w}{\partial x_{j}}\right)^{2}\right] \frac{\varepsilon}{k}-c_{2} \frac{\varepsilon^{2}}{k}
\end{array}\right.
$$

where $k$ is the turbulence kinetic energy; $\varepsilon$ is the turbulence dissipation rate; $K_{m}=c_{\mu} k^{2} / \varepsilon$ is the vertical eddy current coefficient; $c_{\mu}=0.09 ; c_{1}=1.44 ; c_{2}=1.92 ; \sigma_{k}=1.00$ and $\sigma_{\varepsilon}=1.30$.

\subsection{Mud-particle interactions}

During the process of slag removal in the slurry shield machine, the slag particles generate motion due to the effect of the mud. When the particles move along the pipe, the motion will be affected by the particle force acting on the slurry. This will be equal to the surface and the buoyancy forces of the slurry flow acting on the particles based on Newton's third law (note that here we ignore the influence of micro forces such as differential pressure 
force and the virtual mass force of the particle motion). Therefore, when a single particle is not in contact with the pipe wall, there are mainly three forces affect its motion in a pipe, i.e. the force exerted by the slurry, the buoyancy generated by the slurry, and the gravity force of particle itself. However, once the particles contact the pipe wall, there are two additional forces related to the frictional resistance and the collision reaction force against the pipe wall.

The buoyancy force of particles is:

$$
F=\rho g V
$$

where $\rho$ is the density of the mud; $g$ is the acceleration of gravity; $V$ is the mud volume discharged by the particles, respectively.

There is no universal expression of the drag force generated by the muddy flow on the gravel particles. In this study, the formula of drag force suggested by the Di Felice ${ }^{[24]}$ is adopted as follows:

$$
F=\frac{1}{8} C \rho \pi d^{2}\left(U_{f}-U_{p}\right)\left|U_{f}-U_{p}\right| f(\varepsilon),
$$

where $d$ is the diameter of the solid particle; $U_{f}$ is the moving speed of the mud; $U_{p}$ is the moving speed of the solid particle; $\varepsilon$ is the porosity of the solid particle aggregate; $C$ is the drag coefficient, and it is a function of the solid particle Reynolds Coefficient $R_{e}{ }^{[24]}$ :

$$
C=\left(0.63+\frac{4.8}{\sqrt{\mathrm{Re}}}\right)^{2}
$$

where $\operatorname{Re}=\frac{\varepsilon \rho d\left|U_{f}-U_{p}\right|}{\mu}$

In the equation (10), $\mu$ is the hydrodynamic viscosity coefficient. Because of the drag force $F$ formula, $f(\varepsilon)$ is related to the porosity. Due to the large number of particles inside the discharge pipe, there are:

$$
f(\varepsilon)=\varepsilon^{1-m}
$$

where superscript coefficient $m=3.7-0.65 \exp \left[-\frac{(1.5-\operatorname{lgRe})^{2}}{2}\right]$ 
When the slurry flow motion inside the pipe becomes stable, the equation of motion of a single solid particle in the pipe can be written as:

$$
\frac{\pi}{6} d_{e}^{3}\left(\rho_{s}+\frac{\rho}{2}\right) \frac{d v_{s}}{d t}=F_{D}-F_{h}=0
$$

where $F_{D}$ and $F_{h}$ are the drag force of the solid particles from the muddy fluid and other solid particles, and they follow the equations in below:

$$
\begin{gathered}
F_{D}=\frac{\pi}{4} d_{e}^{2} C_{D r} \frac{\left(v_{w}-v_{s}\right)^{2}}{2} \rho \\
F_{h}=\left[1-(1-\bar{q})^{2(n-1)} \frac{\left(\sqrt{\operatorname{Re}} \alpha+\sqrt{\operatorname{Re} \alpha^{2}+4 \sqrt{48} \alpha \beta /(1-\bar{q})^{n-1}}\right)^{2}}{\left(\sqrt{\operatorname{Re} \alpha}+\sqrt{\operatorname{Re} \alpha^{2}+4 \sqrt{48} \alpha \beta}\right)^{2}}\right] W_{b},
\end{gathered}
$$

where $d_{e}$ is the equivalent sphere diameter of the solid particle, $C_{D r}$ is the resistance coefficient based on the velocity difference $v_{W}-v_{s}, R e$ is the particle Reynolds number, $\alpha$ and $\beta$ are the Swanson shape coefficients of the solid particle, $n$ is the prime number, $W_{b}$ is the effective gravity force of the solid particles in water; $W_{b}=\pi d_{e}^{3}\left(\rho_{s}-\rho\right) g / 6$ used in the calculation.

\subsection{Particle-particle and particle-pipe wall interactions}

The combination effect of the particle motion, the particle-particle collision, the particle and the pipe wall interaction will affect the particle moving into the muddy fluid of the surrounding continuous phases. The energy transfer and the momentum transport are all simulated using the discrete element method. As the particle-particle collision and the particle/wall surface interaction do not cause significant plastic deformation of the particle itself, we consider either a hard particle contact model ${ }^{[25-26]}$ and/or a 'wet' particle contact model ${ }^{[27]}$. The interaction between particle-particle and particle-pipe wall must also satisfy the Newton's second law. Within this context, while the particles are moving inside the pipe, the surface of the particles must adhere with the surrounding muddy fluid. As a result, the surface of the particles becomes sticky. Therefore, the JKR variant of the Hertz-Mindlin Cohesion model ${ }^{[28]}$ is adopted for the calculation of particle-particle collision, and the particle and 
the Hertz-Mindlin (no-slip) method is employed for the calculation of the particle and the pipe wall interaction. The former was chosen to simulate viscous effect of particles in the pipe because it is a cohesive contact model. It can consider the influence of Van der Waals forces in the contact area and allow the user to simulate fluids of high viscosity. The calculation is made by evaluating the tangential elastic force, the normal dissipation force and the tangential dissipating force, respectively.

The normal force is based on the overlapped amount $\delta$, the interaction parameter $\alpha$ and the surface energy $\gamma$ as follows

$$
\begin{gathered}
F_{J K R}=-4 \sqrt{\pi \gamma E^{*}} \alpha^{3 / 2}+\frac{4 E^{*}}{3 R^{*}} \alpha^{3} \\
\delta=\frac{\alpha^{2}}{R^{*}}-\sqrt{\frac{4 \pi \gamma \alpha}{E^{*}}}
\end{gathered}
$$

where $E^{*}$ is the equivalent Young's modulus and $R^{*}$ is the equivalent radius of the particle.

In the model, when $\gamma=0$, the normal force has a simpler form as :

$$
F_{\text {Hertz }}=\frac{4}{3} E^{*} \sqrt{R^{*}} \delta^{\frac{3}{2}}
$$

This model provides attractive cohesion, even if the particles are not in direct contact. The maximum gap between particles with non-zero cohesive force is calculated by the following equations:

$$
\begin{aligned}
\delta_{c} & =\frac{\alpha_{c}^{2}}{R^{*}}-\sqrt{\frac{4 \pi \gamma \alpha_{c}}{E^{*}}} \\
\alpha_{c} & =\left[\frac{9 \pi \gamma R^{* 2}}{2 E^{*}}\left(\frac{3}{4}-\frac{1}{\sqrt{2}}\right)\right]^{\frac{1}{3}}
\end{aligned}
$$

When the condition of $\delta<\delta_{c}$ is satisfied, the model recovers to its original format. When the particles are not actually in direct contact and the spacing of the gap is less than $\delta_{c}$, the cohesive force reaches a maximum value. This maximum cohesion force, also called pull-outface, is:

$$
F_{\text {pullout }}=-\frac{3}{2} \pi \gamma R^{*}
$$


Additionally, the Hertz-Mindlin (no-slip) model is used to predict for the interaction of the pipe wall and the particles. In this model, the normal force between the particle and the pipe wall is:

$$
F_{n}=\frac{4}{3} E^{*} \sqrt{R^{*}} \delta_{n}^{\frac{1}{3}}
$$

where $\frac{1}{E^{*}}=\frac{\left(1-v_{1}^{2}\right)}{E_{1}}+\frac{\left(1-v_{2}^{2}\right)}{E_{2}}, \frac{1}{R^{*}}=\frac{1}{R_{1}}+\frac{1}{R_{2}} . E^{*}$ is the equivalent Young's modulus; $R^{*}$ is equivalent radius; $E_{1}, E_{2}$ are Young's modulus of the particle and the pipe ; $V_{1}$ and $V_{2}$ are the velocity of two particles in contact; $R_{1}$ and $R_{2}$ are the radius of the particles in contact, respectively.

The expression of the normal damping force is:

$$
F_{n}^{d}=-2 \sqrt{\frac{5}{6}} \beta \sqrt{S_{n} m^{*}} v_{n}^{\overline{r e l}},
$$

where $m^{*}=\left(\frac{1}{m_{1}}+\frac{1}{m_{2}}\right)^{-1}$ is the equivalent mass, $v_{n}^{\frac{1}{r e l}}$ is the normal component of the relative velocity between the particle and the pipe, $\beta$ and $S_{n}$ are defined as:

$$
\begin{gathered}
\beta=\frac{\ln e}{\sqrt{\ln ^{2} e+\pi^{2}}} \\
S_{n}=2 E^{*} \sqrt{R^{*} \delta_{n}}
\end{gathered}
$$

where $e$ is the coefficient of restitution. Tangential force $F_{t}$ depends on tangential overlap and tangential stiffness:

$$
F_{t}=-S_{t} \delta_{t}
$$

where $S_{t}=8 G^{*} \sqrt{R^{*} \delta_{n}}$.

The parameter $G^{*}$ is the equivalent shear modulus. The tangential damping force is:

$$
F_{t}^{d}=-2 \sqrt{\frac{5}{6}} \beta \sqrt{S_{t} m^{*}} v_{t}^{\overline{r e l}}
$$

where $v_{n}^{\overline{r e l}}$ is the tangential component of relative velocity.

For the rolling friction, Hertz-Mindlin (no-slip) method is considered by applying a shear moment to the contact surface as $\tau=-\mu_{r} F_{n} R \omega$, in which $\mu_{r}$ is the rolling friction coefficient; $R$ is the distance from the contact 
point to the centroid of particle, $\omega$ is the unit acceleration vector of the particle at the contact point, respectively.

\subsection{CFD-DEM coupling process}

For the solid and flow coupling process, the first step is to calculate the flow field by CFD model via Fluent solver. Once the flow field converges to a steady-state, the flow field data are imported to the EDEM code through the I/O interface. Then, the EDM model applies flow forces predicted by CFD simulation to the particles and compute the particle motion and trajectory by the EDEM code. Once the EDEM calculation is completed, the information of particle's new position, velocity, and acceleration will be transmitted back to the ANSYS Fluent through the I/O interface. The Fluent software calculation continues based on these received EDEM data. At this point, the particle information are used as a sink source in the momentum equation for the fluid flow. This cycle continues until the coupling calculation is completed eventually ${ }^{[31]}$ (see, e.g. Figure 1).

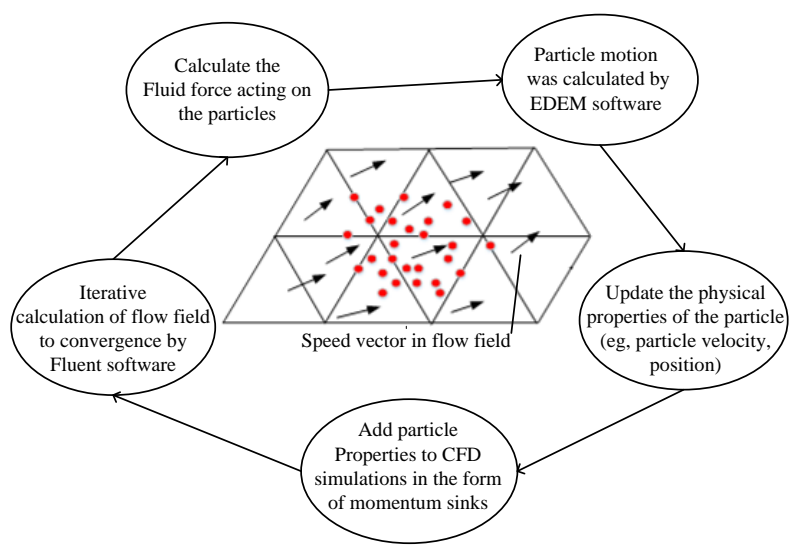

Figure 1 CFD-DEM coupling calculation process.

\section{Pipe model and parameter settings}

\subsection{Pipe model}

The pipe adopts two transitional forms. The pipe diagram is shown in Figure 2, where $G$ is the direction of gravitational acceleration. 


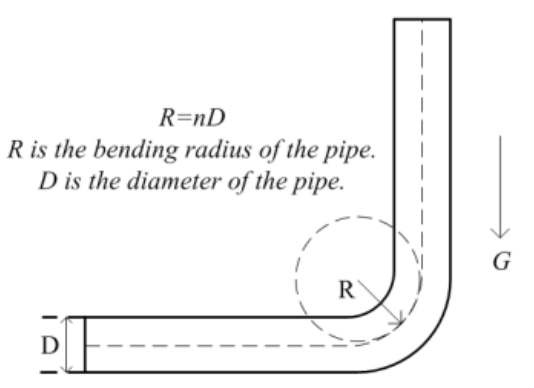

(a) Circular transition curve

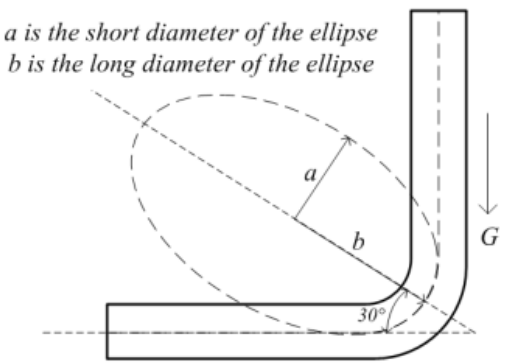

(b) Elliptic transition curve

Figure 2 Schematic diagram of the pipe illustrating (a) circular pipe; (b) Elliptic pipe.

In the process of pipe pulping, the direction changes of the pipe are essential for flow movement. When the pipe tuning angle is fixed at $90^{\circ}$, the variation of the bending radius of the pipe will have impacts on the motion of particles inside the pipe. To evaluate and compare, this study considers the bending radius of 1 time, 2 times, 3 times, 4 times, 5 times and 6 times of the pipe diameter, respectively. Figure 3 illustrates a sketch of 3D model of the pipes.

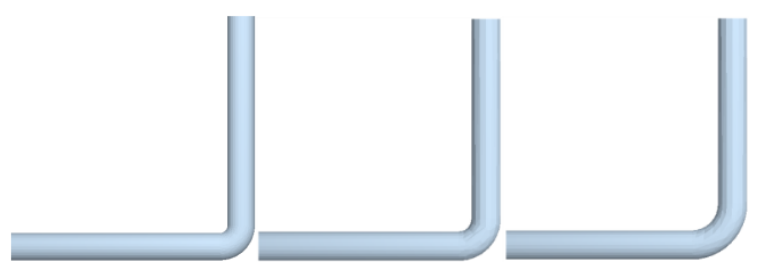

(a) $0.3 \mathrm{~m}$ bend

(b) $0.6 \mathrm{~m}$ bend

(c) $0.9 \mathrm{~m}$ bend

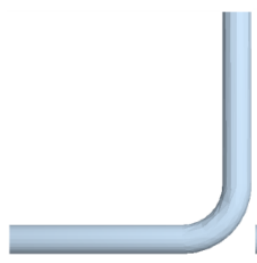

(d) $1.2 \mathrm{~m}$ bend

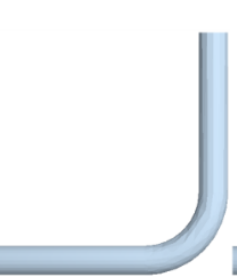

(e) $1.5 \mathrm{~m}$ bend

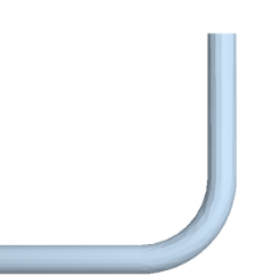

(f) $1.8 \mathrm{~m}$ bend

Figure 3 The pipe bending diameter is an integer multiple of the pipe diameter.

When the muddy fluid is flowing inside the pipe, the fluid velocities will alter due to the change of the pipe direction. Due to inertia, particles in the pipe will have tendency to continue to move along the original direction, 
and thus particles will inevitably collide with the pipe wall. To minimize this effect, the pipe can be designed using a transitional bending shape, so that the direction of the slurry flow in the pipe can be gradually changed along the bend. This will further influence the velocity vector of the particles in the pipe to avoid drastically direction change. Overall, this will reduce the collision of the particles with the pipe wall. Figure 4 shows a sketch of a 3D pipe model with different transitional bends of elliptic shape.

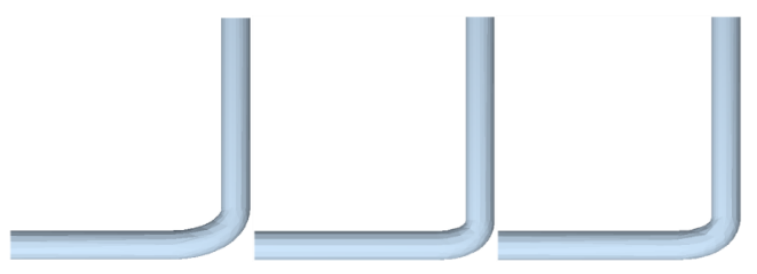
(a) $0.5 \mathrm{~m}-0.9 \mathrm{~m}$
(b) $0.5 \mathrm{~m}-1.2 \mathrm{~m}$
(c) $0.6 \mathrm{~m}-1.5 \mathrm{~m}$

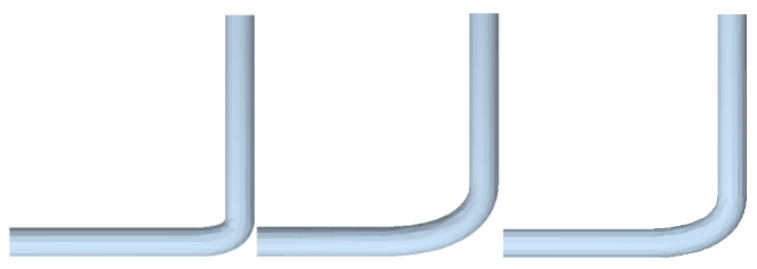

$\begin{array}{lll}\text { (d) } 0.6 \mathrm{~m}-1.8 \mathrm{~m} & \text { (e) } 0.9 \mathrm{~m}-1.8 \mathrm{~m} & \text { (f) } 0.9 \mathrm{~m}-2.1 \mathrm{~m}\end{array}$

Figure 4 The curve of the pipe is an elliptic transition curve.

\subsection{Physical parameters of the gravel particles}

Based on analysis of onsite samples collected from the actual tunneling places, a set of the particles and other physical parameters are defined in the following Table 1 . These parameters will be used in the calculation unless otherwise stated.

Table 1 Basic parameters

Physical properties Symbol Unit Value 


\begin{tabular}{|c|c|c|c|}
\hline Particle density & $\rho_{0}$ & $\mathrm{Kg} / \mathrm{m}^{3}$ & 1500 \\
\hline $\begin{array}{l}\text { Particle-particle static } \\
\text { friction coefficient }\end{array}$ & $f$ & 1 & 0.3 \\
\hline $\begin{array}{c}\text { Young's Modulus of } \\
\text { Particles }\end{array}$ & $Y_{0}$ & $\mathrm{~Pa}$ & $1.2 \mathrm{e}+10$ \\
\hline Poisson's ratio of particles & $\mu_{0}$ & 1 & 0.25 \\
\hline Density of the pipe & $\rho_{1}$ & $K g / m^{3}$ & 7800 \\
\hline $\begin{array}{c}\text { Young's modulus of pipe } \\
\text { material }\end{array}$ & $Y_{1}$ & $P a 2$ & $2.0 \mathrm{e}+10$ \\
\hline Poisson's ratio of pipe & $\mu_{1}$ & 1 & 0.3 \\
\hline $\begin{array}{l}\text { Particle-particle dynamic } \\
\text { friction coefficient }\end{array}$ & $\lambda_{0}$ & 1 & 0.2 \\
\hline $\begin{array}{c}\text { Particle-particle recovery } \\
\text { factor }\end{array}$ & $e_{1}$ & 1 & 0.01 \\
\hline $\begin{array}{l}\text { Particle-pipe static friction } \\
\text { coefficient }\end{array}$ & $\lambda_{1}$ & 1 & 0.2 \\
\hline $\begin{array}{l}\text { Particle-pipe dynamic } \\
\text { friction coefficient }\end{array}$ & $\lambda_{2}$ & 1 & 0.545 \\
\hline Particle-pipe recovery factor & $e_{2}$ & 1 & 0.01 \\
\hline Particle surface energy & $K$ & $J$ & 5 \\
\hline Gravity acceleration & $G$ & $m / s^{2}$ & 9.81 \\
\hline Rate of particle generation & $n$ & 1 & 2000 \\
\hline
\end{tabular}

\section{Simulation Results and Analysis}

To simulate the flow motion of gravel and mud mixture, the following assumptions are made, i.e. to ignore the pipe pressure loss, mainly because only a small section of the pipe is taken for investigation. This means the pressure along the pipe is constant at 5 bar and the flow rate is $80 \mathrm{~m}^{3} / \mathrm{h}$.

In case of different pipe bending radii and shapes, the production speed and the shape of the particles are also the same, that is, the shape and the numbers of particles that enter the pipe within a unit of time are the same. The 
physical properties of the slurry in the pipe and the initial velocity of the slurry are also the same. Under these conditions, the number of colliding particles, the number of contacts, the mass of the particles, the tangential energy loss of the particles, the velocity of the muddy fluid in the pipe, the total pressure, and the turbulence intensity of the mud will be compared and analyzed under different pipe shape conditions, in order to determine the influence of the pipe bending radii and shape on the motion of the slurry and the particles, and also their impact on the pipe wear and tear.

\subsection{Particle motion characteristics analysis}

Because of the same production rate of particles in the pipe and the physical properties of the particles, the boundary conditions of the slurry flow in the pipe are kept the same. Thus, the effect of the pipe bending shape change on the performance of the pipe slag discharge can be analyzed by comparing the motion characteristics of the particles after the flow passing the pipe bend. Figure 5 gives predicted mass in relation to the pipe bend of elliptic curve, e.g. 0.5-0.9 representing the minor diameter of the transitional ellipse with a diameter of $0.5 \mathrm{~m}$ and the major diameter of $0.9 \mathrm{~m}$. This definition also applies for other five bending pipes, respectively. For the pipe bend with circle shape, as the inner diameter of the pipe is 0.3 meters, 1 time the diameter of the pipe means that the diameter of the transition circle of the pipe at the turn is 0.3 meters, and 2 times the diameter of the pipe means that the diameter of the transition circle of the pipe at the turn is about 0.6 meters. The following is the predicted motion characteristics of the particles in the pipe for several selected cases. 


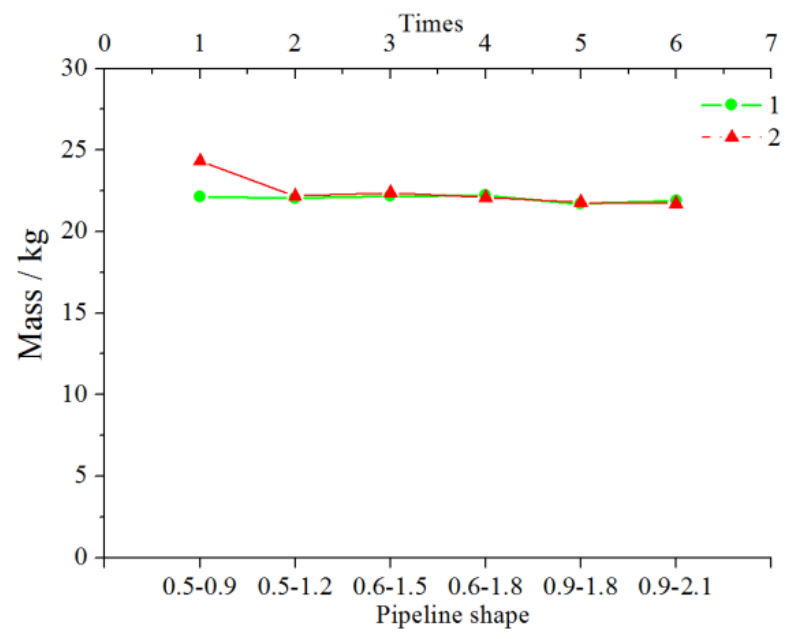

Figure 5 The total mass of the particles in the pipe.

Figure 5 shows the predicted total mass of particles in the pipe after the fluid flow is converged under different pipe shapes. Curve 1 indicates the mass of the particles in the pipe at different transitional bends (i.e. elliptic bending shape, note same notation used later), and curve 2 indicates the mass of the particles in the pipe at a multiple of the diameter of the different pipes (i.e. circle bending shape, note same notation used later). It can be seen from curve 1 that when the transition radius is larger than the pipe diameter, the mass of the particles in the pipe has little change after the fluid flow being 'stabilized' in the pipe. For curve 2, when the diameter of the pipe is 1 time the diameter of the pipe, the mass of the particles in the pipe reaches the maximum; When the diameter of the pipe is no less than twice of the diameter of the pipe, the mass of the particles in the pipe has little change with the increase of the pipe diameter.

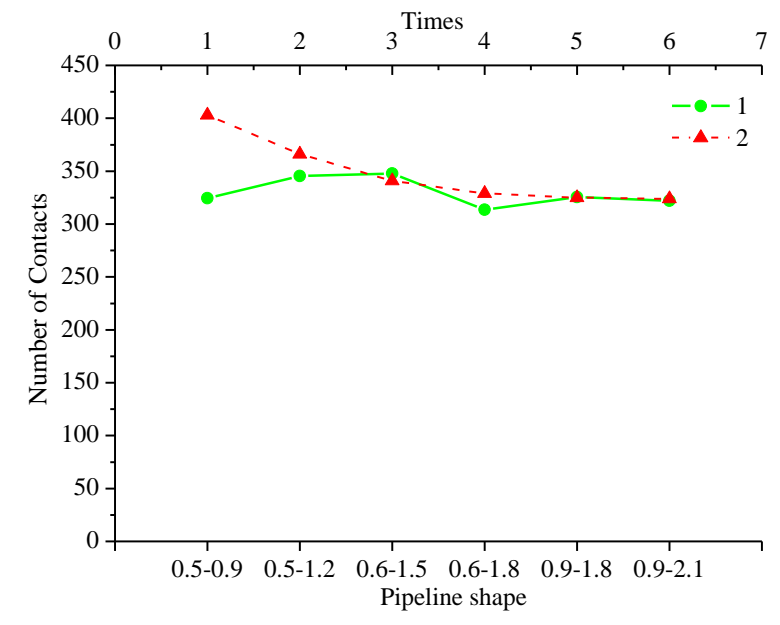


Figure 6 The number of particles and pipe contact.

Figure 6 shows the number of contacts between the particles and the pipe wall for different pipe shapes when the fluid flow is stabilized and. It can be seen from curve 1 that while the ratio of the major to the minor axes of the ellipse shape increases, the number of contacts between the particles and the pipe wall has small variations. For Curve 2, the number of contacts between the particles and pipe walls in the pipe shows a gradual decrease until the point with four times of the diameter, then it becomes nearly constant.

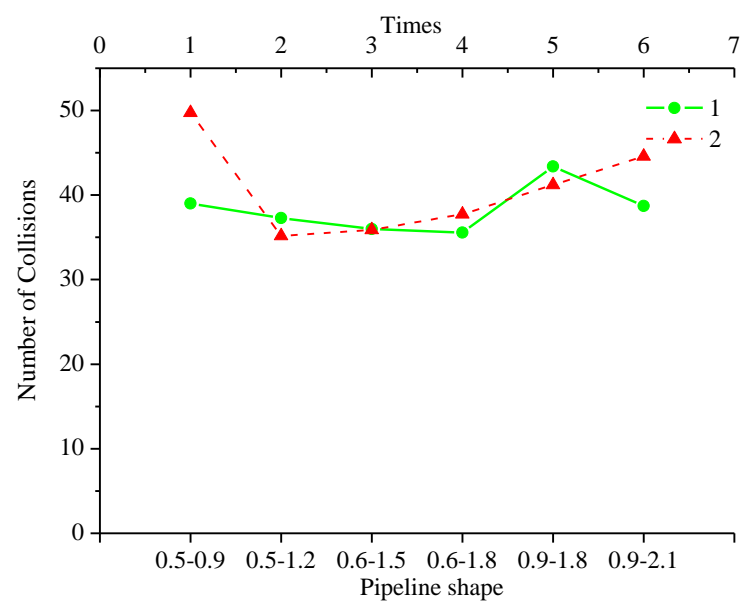

Figure 7 Collisions between particles and pipes.

Figure 7 shows the number of collisions between gravel particles and with the pipe wall in the pipe after the fluid flow being stabilized. The curve with correspondent to pipeline shape over a number of particle collision has shown a decrease and increase tendency. For the curve 1, it was seen that the number of contacts between the particles and the pipe wall firstly increases and then decreases, as the ratio of the major to the minor axes of the ellipse shape increases. When ratio of major and minor diameters of the ellipse is 2 (i.e. 0.9-1.8 case), the number of collisions reaches a maximum value of [42.5?]. For Curve 2, as the bending radius of the pipe is one time of the pipe diameter, the number of collisions reaches a maximum value of 50 . This follows a sudden drop to minimum value of 35 when the pipe bending radius is two times of the pipe diameter. After that, the number of collisions increases steadily with the increase of the pipe bending radius. Therefore, it is concluded that when the 
bending radius of the pipe is 2 times of the diameter of the pipe, the collision between the particles and the pipe wall reaches the minimum level, which is beneficial to reduce the collision damage of the particles to the pipe wall.

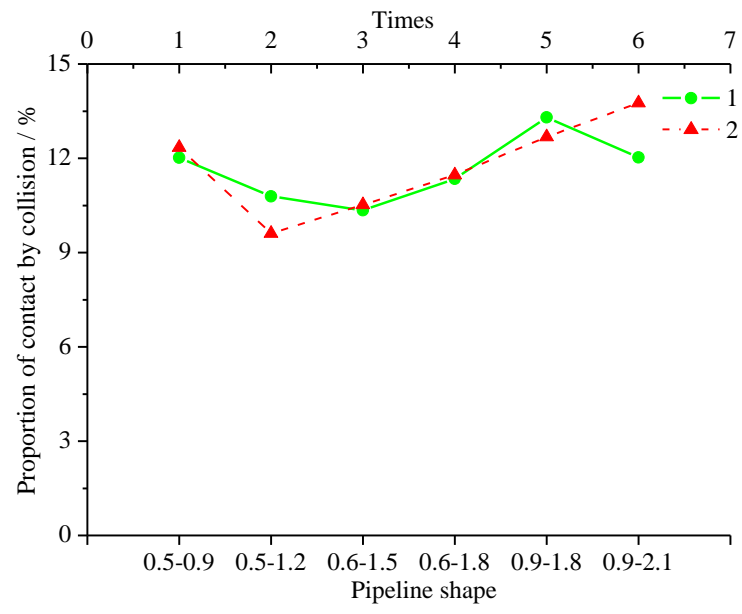

Figure 8 The ratio of the number of collisions to the number of contacts.

Figure 8 shows the ratio of the number of collisions and the number of contacts between the particles and the pipe wall. For curve 1 and curve 2, the ratio of the number of collisions to the number of contacts shows a broad tendency of decreasing and then increasing features. For curve 1, when the transition radius of the pipe is 0.6-1.5, the ratio of the number of collisions and contacts is the smallest. For curve 2, when the bending radius of the pipe is 2 times of the diameter of the pipe, the ratio of the number of collisions between the particles and the pipe wall has the lowest value. Subsequently, the ratio increases with the increase of bending radius. 


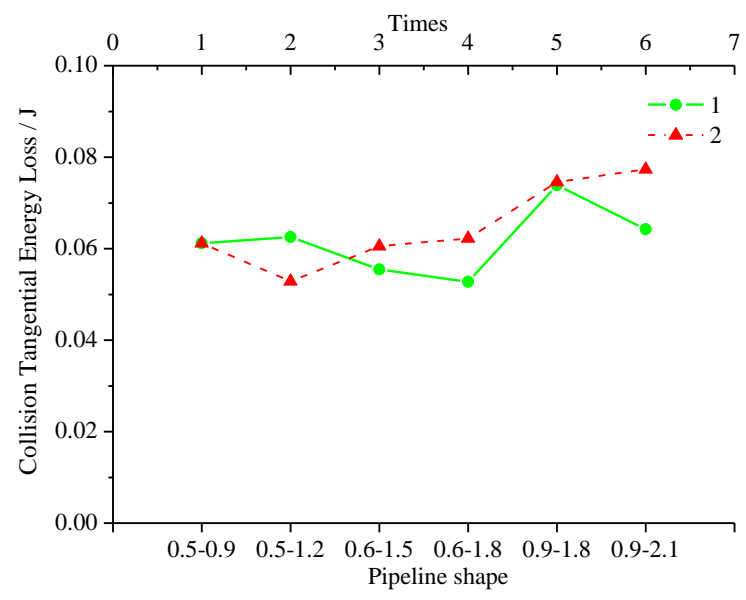

Figure 9 Loss of tangential energy in collisions between particles and pipes.

Figure 9 shows the tangential energy loss due to the collisions between particles and the pipe walls after the flow of muddy fluid in the pipe being stabilized. During the collision process, these tangential energy losses of the particles are converted into the work done by the friction between the particles and the pipe wall, and finally reflected by the wear and tear of the pipe walls. The more the tangential energy loss of the particles is, the greater the frictional wear/tear on the pipe walls would be. From the curve 1, it can be seen that as the ratio of the major to the minor axes of the ellipse shape increases, the tangential energy loss firstly increases and then decreases. When this ratio is 2 (i.e. 0.9-1.8 case), the tangential energy loss reaches a maximum value. For Curve 2, when the pipe bending radius is 2 times of the diameter of the pipe, the tangential energy loss of the particles is the smallest; when the pipe bending radius increases, the tangential energy loss increases accordingly.

\subsection{Analysis of the motion characteristics of slurry flow}

\subsubsection{The motion of slurry flow for the bending radius of the pipe with circle shape}



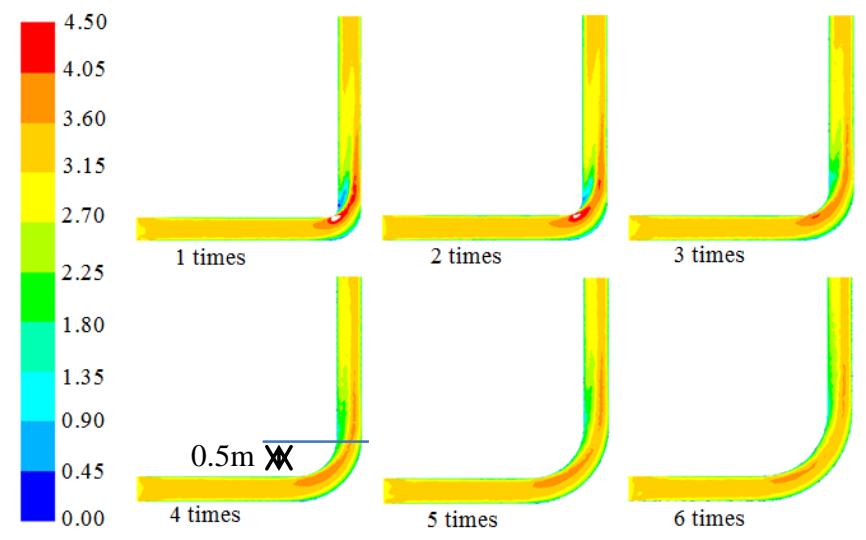

(a) Middle section of the pipe
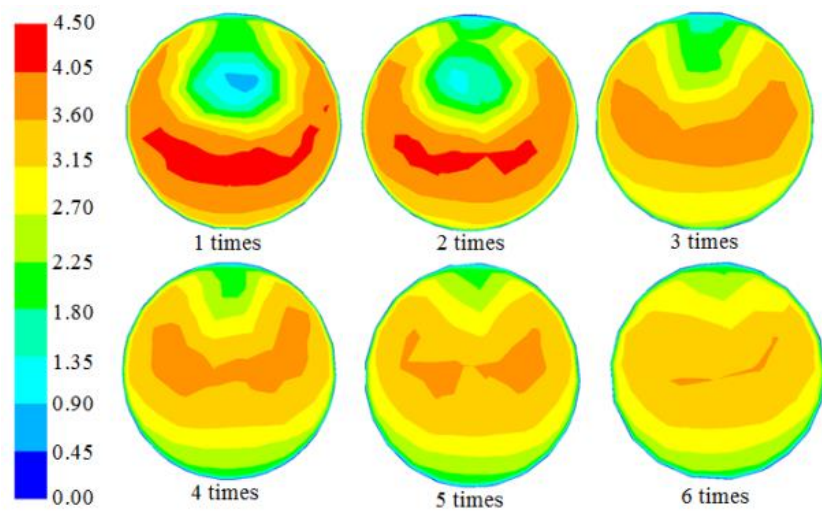

(b) Cross section of the pipe

Figure 10 Flow velocity contours of mud fluid in the pipe $(\mathrm{m} / \mathrm{s})$.

Figure 10(a) shows the acceleration of muddy fluid flowing along the near-field of the bending pipe wall, evidenced by contours in red color scale. With the increase of the bending radius of the pipe, the velocity in the near-field of the pipe bends reduces; indicating mass flow rate of the mud reduces. From the velocity contours at a cross section of the pipe, it can be seen that the velocity of mud flowing through the pipe bend has a very large gradient when the bending radius of the pipe is one time of the diameter of the pipe. This rapid change between high-speed and low-velocity are formed around the center of the pipe. When the bending radius of the pipe is 2 times of the diameter of the pipe, the difference between the high-speed and the low-speed flow becomes slightly less, and the trend of decreasing continues with the increase of the bending radius of the pipe. As a result, the 
scouring effect of mud in the pipe will be reduced with the increase of bending radius, which is beneficial to prolong the life of the pipe.

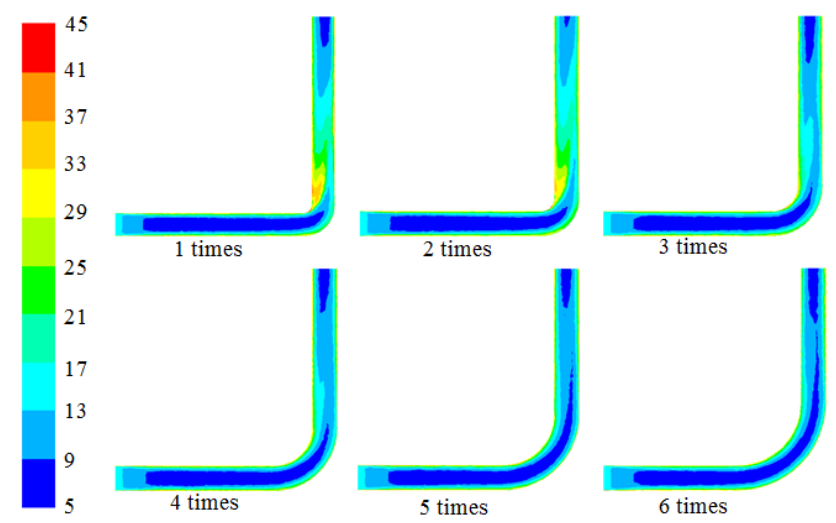

(a) Middle section of the pipe

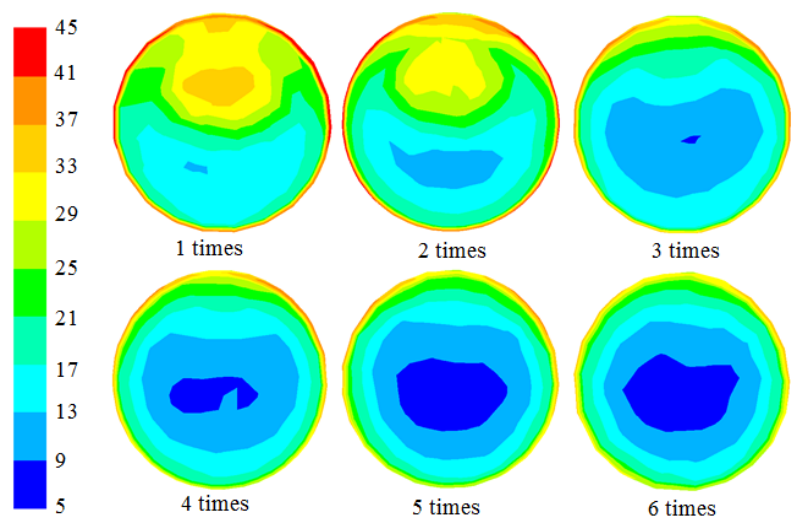

(b) Cross section of the pipe

Figure 11 Turbulence intensity (\%) contours of mud fluid in the pipe.

From Figure 11(a), turbulence intensity is weak initially at the pipe entry the turbulence intensity increases to maximum at the bend for pipe bending radius having 1 time or 2 times of the pipe diameter. The turbulence intensity becomes weakest for the pipe with bending radius larger than 3 times of the pipe diameter. From the cross-sectional contours (i.e. Fig. 11(b)), the turbulence intensity of the mud at the near pipe wall region in the circumferential direction is greater for all cases. However, when the bending radius of the pipe is 1 time and 2 times of the diameter of the pipe, there are large areas in the near-field of the bends covered with higher 
turbulence intensity. This is due to the presence of fast-moving high-speed fluid. With the increase of the bending radius of the pipe, the turbulence intensity of the mud in the pipe is weakened in further. As the turbulence intensity is an indicator of the level of velocity fluctuations, high turbulence intensity means that rapid change of flow direction (such as 1 time or 2 times of bending radius) may lead to high shear around the bend. This high shear will trigger high level of flow fluctuations, echoed by high turbulence intensity level.

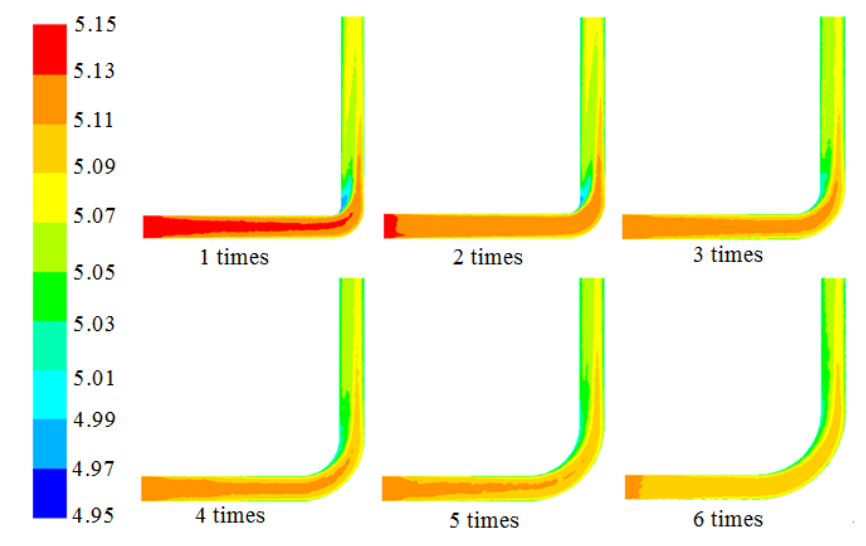

(a) Middle section of the pipe
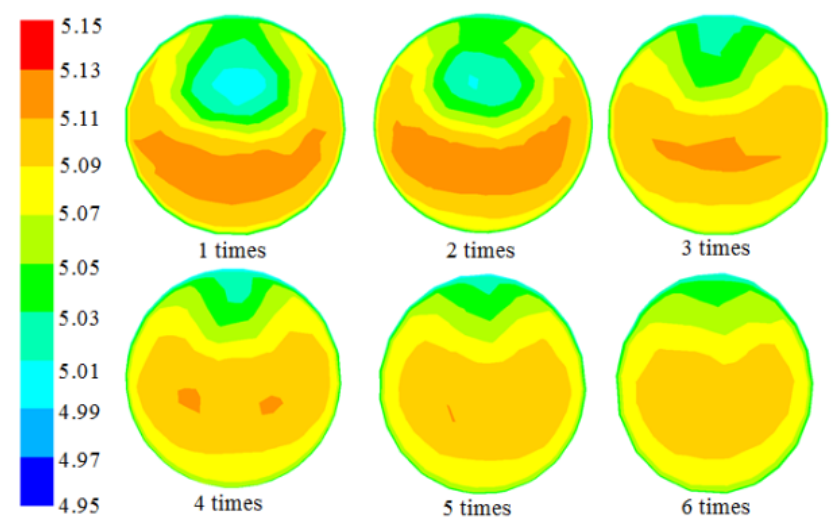

(b) Cross section of the pipe

Figure 12 Total pressure contours of mud in the pipe (bar).

It can be seen from Figure 12 that with the increase of the pipe bending radius, the total pressure of the fluid in the pipe decreases, indicating energy loss possibly converting to heat or other means. It can be seen from the cross-section that with the increase of the bending radius of the pipe, the change of the total pressure gradient due 
to the mud passes through the bend is reduced gradually. , The higher total mud pressure is observed to tends to concentrate in the pipe center for those pipes with larger bending radius, indicating an extended the life of the pipe.

\subsubsection{Analysis of Slurry Movement in the Pipe under Transitional (Elliptic) Curve}

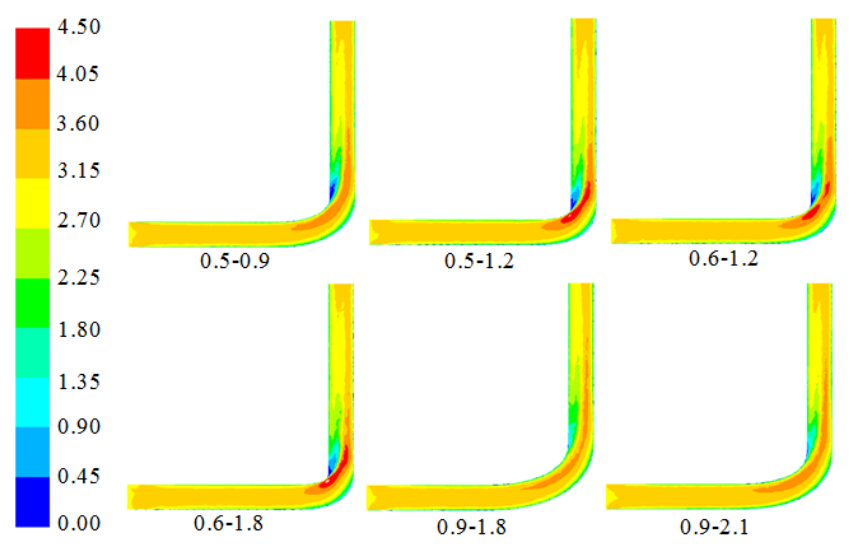

(a) Middle section of the pipe
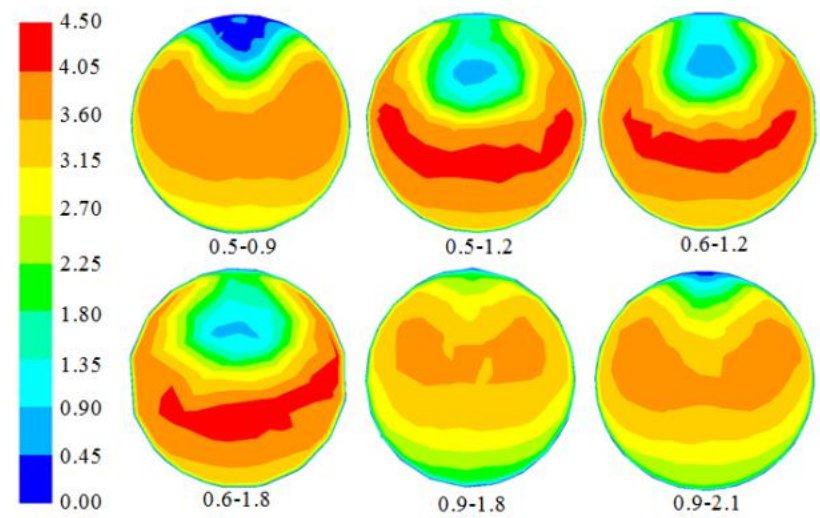

$0.5-1.2$

$0.6-1.2$
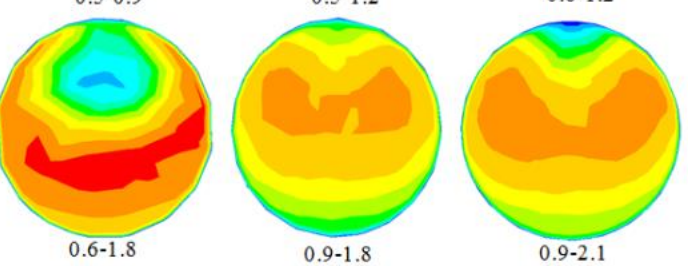

(b) Cross section of the pipe

Figure 13 Velocity of mud in the pipe $(\mathrm{m} / \mathrm{s})$.

It can be seen from Figure 13(a) that for all six cases of transitional bends, the smoother the transition of the pipe around the bending cross section, the smaller the gradient of the velocity gradient of the mud in the pipe. 
From the cross section of the pipe (i.e. Fig. 13(b)), it can be seen that the gradient of the fluid velocity in the pipe is the smallest compared to other five cases when the transition ellipse shape pipe having a radius of 0.9-1.8, indicating that the erosion of the slurry by the mud would be less.

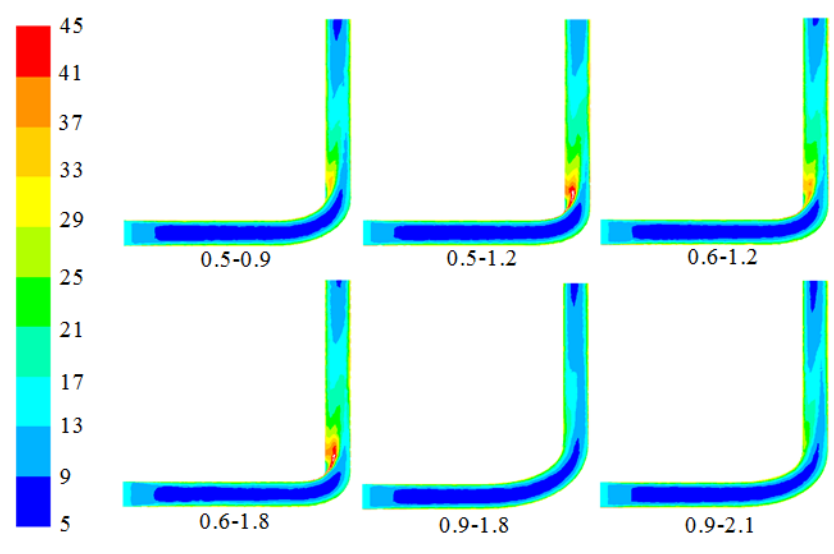

(a) Middle section of the pipe

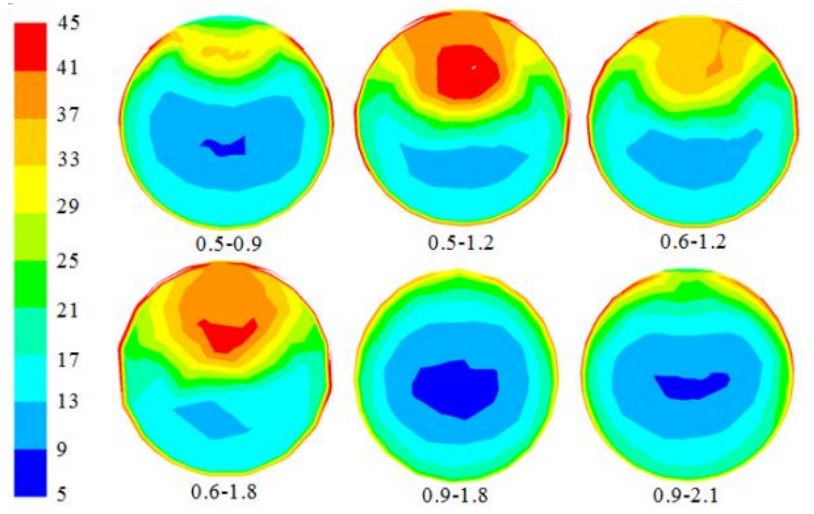

(b) Cross section of pipe

Figure 14 Turbulence intensity (\%) contours of mud in the pipe.

Figure 14 shows the turbulence intensity of mud fluid for the cases of transitional curved bends. While in comparing Figure 14 and Figure 13, it can be seen that in the region where the velocity of the mud fluid in the pipe is smaller, the turbulence intensity is higher, and vice versa. 


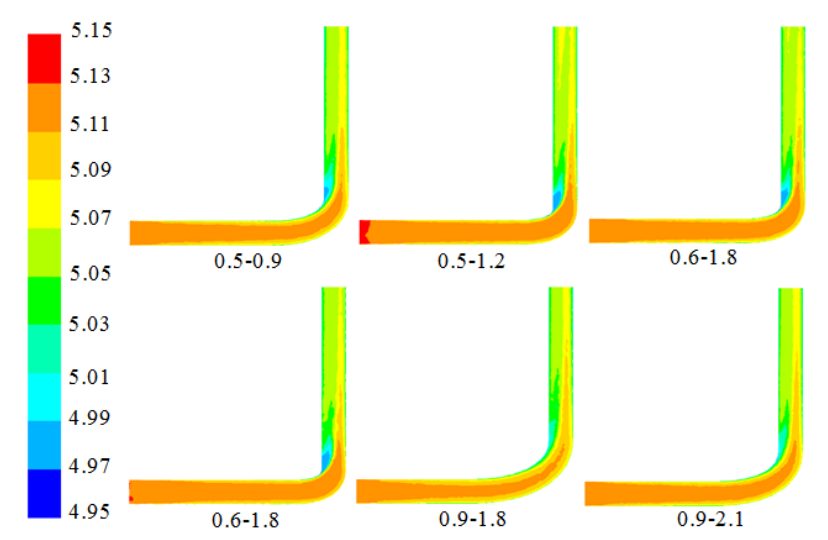

(a) Middle section of the pipe
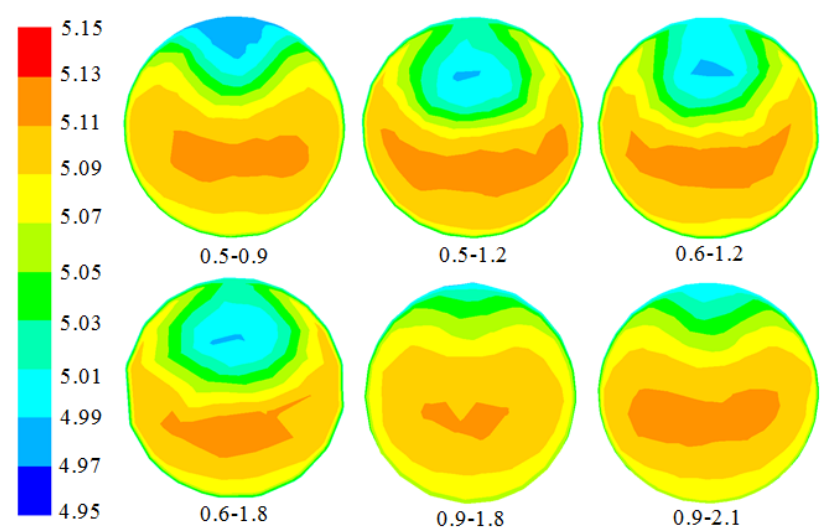

(b) Cross section of the pipe

Figure 15 Total pressure of mud in the pipe (bar).

From Figure 15, it can see that for the case with transitional curve of $0.9-1.8$, the total pressure variation in the pipe is relatively small, compared to other five transitional cases, indicating less total pressure loss for this configuration. From Fig. 15(b), when the pipe transition ellipse curve is $0.9-1.8$, the total pressure has shown less variation cross the pipe, in consistent with that seen in Fig. 15(a).

By comparing and analyzing the motion characteristics of the particles in the above 12 kinds of pipes, it can be known that increasing bending-diameter ratio of the pipe can't reduce the collision between particles and the pipe, and reduce the pipe wear, but it can weaken the mud turbulence in the pipe. Under the initial conditions of this paper, when the pipe diameter to diameter ratio is 2 , it has shown the most beneficial to prolong the life of the pipeline, which is beneficial to the discharge of particles in the pipeline. When the ratio of the long diameter to the 
short diameter of the ellipse is 2 , the collision of the particles on the pipeline is the most severe, which is unfavorable for prolonging the life of the pipeline and has little effect on the slagging performance. Such a pipeline structure should be avoided.

\section{Conclusions}

Based on the comparison and analysis of the movement characteristics of particles and mud flow in six different bending radii and six different bending shapes of a $90^{\circ}$ tuning pipe using CFD-DEM methodology, the following conclusions can be drawn:

For a circular bending shape, when the pipe bending radius is 2 times of the pipe diameter, this pipe configuration will be the most conducive in terms of the discharge of slag within the pipe, which can reduce the wear and tear of the pipe walls and extend the service life of the pipe.

For an elliptic bending shape, when the ratio of the major to the minor axes of a transitional curve of the pipe is 2, this pipe configuration will be the least conducive in terms of the discharge of the slag within the pipe. This layout should be avoided when designing the pipe bends.

Due to the coexistence of viscous mud and slag particles in the pipe, the movement of mud and scum in the pipe could be more complicated in real applications. In fact, the slag particles move under the combined influence of gravity, buoyancy, inertia forces of the muddy, in addition to high turbulence trigger over the rapid bending area. Through the simulations presented in this study, the relation between the bending radius of the pipe that is the most beneficial to the pipe slagging, and the flow characteristics has been obtained quantitatively and qualitatively, which lays the solid foundation for further investigations. The future work could be theoretical studies to illustrate the particle movement mechanism under the effect of viscosity of the mud, which will also help to reveal the interactions between the particle motion and the muddy turbulence flow. 


\section{Acknowledgements}

This research was supported by China Railway Tunnel Stock CO., LTD (Contract no. to add here if you have), who is also providing the physical parameters of slurry and particles used in this study.

\section{References}

[1] Junzhou Huo, Bao Cai, Yajie Wang, Xiang-yu OuYang. The Mechanical Analysis and Optimal Design Support Ribs of Full-Face

Tbm Based on Dalian Subway Project. Machinery Design \& Manufacture, 2014, 10: 57-60.

[2] Binbin Li, Jian Du, Wen Liu. Mechanical Analysis of the Cutter Head of an EPB Shield. Mechanical Research \& Application. 2017, 1: 32-34.

[3] Hu Shi, Huayong Yang, Guofang Gong, Lintao Wang. Determination of the Cutterhead Torque for EPB Shield Tunneling Machine. Automation in Construction, 2011, 4(20): 1087-1095.

[4] Geng Qi, Zhengying Wei, Hao Meng, Chen Qiao. Numerical and Experimental Research on the Rock-breaking Process of Tunnel Boring Machine Normal Disc cutters. Journal of Mechanical Science and Technology, 2016, 4: 1733-1745.

[5] Sayed Rahim Moeinossadat, Kaveh Ahangari, Kourosh Shahriar. Calculation of Maximum Surface Settlement Induced by EPB Shield Tunnelling and Introducing most Effective Parameter. Journal of Central South University of Technology, 2016, 23(12): 3273-3283.

[6] Caihui Zhu, Ning Li. Prediction and Analysis of Surface Settlement Due to Shield Tunneling for Xi'an Metro. Canadian Geotechnical Journal, 2016, 54 (4):529-546.

[7] Xingchun Li, Xinggao Li, Dajun Yuan, Yuhai Guo. Using Least Squares Support Vector Machine to Predict the Maximum Ground Surface Settlement Caused by Shield Tunneling. Electronic Journal of Geotechnical Engineering, 2017, 22: 613-626.

[8] Yoshihiro Ooishi, Shinichi Murakawa, Akira Hatakoshij, Teruyuki Mori. Development of Discharged Soil Measuring device for Shield Tunneling Machines. Journal of Terramechanics, 1992, 29: 465-475.

[9] Hu Shi, Huayong Yang, Guofang Gong, Huaiyin Liu, Dianqing Hou. Energy Saving of Cutter-head Hydraulic Drive System of 
Shield Tunneling Machine. Automation in Construction, 2014, 37: 11-21.

[10] Hu Shi, Guofang Gong, Huayong Yang, Xuesong Mei. Energy Saving Analysis of Segment Positioning in Shield Tunneling Machine Considering Assembling Path Optimization. Journal of Central South University of Technology, 2014, 21(12): $4526-4536$.

[11] Xu Yang, Guofang Gong, Huayong Yang, Lianhui Jia, Qunwei Ying. A Cutter-head Energy-saving Technique for Shield Tunneling Machines Based on Load Characteristic Prediction. Journal of Zhejiang University, 2015, 16(5): 418-426.

[12] Huayong Yang, Hu Shi, Guofang Gong, Guoliang Hu. Electro-hydraulic Proportional Control of Thrust System for Shield Tunneling Machine. Automation in Construction, 2009, 18: 950-956.

[13] Huayong Yang, Hu Shi, Guofang Gong. Motion Control of Thrust System for Shield Tunneling Machine, Journal of Central South University of Technology, 2010, 17: 537-543.

[14] Lintao Wang, Guofang Gong, Hu Shi, Huayong Yang. Modeling and Analysis of Thrust Force for EPB Shield Tunneling Machine. Automation in Construction, 2012, 27: 138-146.

[15] LinTao Wang, Wei Sun, GuoFang Gong, Huayong Yang. Method to Avoid the Structural Interference of the Thrust System of a Shield Tunneling Machine. SCIENCE CHINA: Technological Sciences, 2017, 60(5): 701-712.

[16] Qian Zhang, Chuanyong Qu, Zongxi Cai, Yilan Kang, Tian Huang. Modeling of the Thrust and Torque Acting on Shield Machines During Tunneling. Automation in Construction, 2014, 40: 60-67.

[17] Yong Zhao, Hao Pan, Hao Wang, Haidong Yu. Dynamics Research on Grouping Characteristics of a Shield Tunneling Machine's Thrust System. Automation in Construction, 2017, 76: 97-107.

[18] Bangjiang Qin, Yimin Xia. The Differential Pressure Analysis and Calculation of Pipeline Circulation System in Slurry Shield. Hydraulics, Pneumatics \& Seals, 2016, 7: 52-55.

[19] Nannan Zhai, Weishan Wang, Baicun Zheng. Rheological Properties of Mud Slurry for Slurry Shield. Chinese Journal of Underground Space and Engineering, 2017, 4(13): 58-64.

[20] Yumeng Bie, Jun Yuan, Jian Cheng, Fuqing Zhang, Xingxing Tang. Formation Mechanism and Solution of Shield Machine Mud 
Block. Engineering Material \& Equipment, 2017, 3(02): 181-184.

[21] Borang Dong. Countermeasures for Reducing Wear and Vibration of Slurry Pipe of Slurry Shield. Tunnel Construction, 2016, 36(11): 1385-1388.

[22] Hai Wang, Xiaochun Zhong. Study on Influence of Muck Screening Efficiency on Slurry Property in Slag Slurry Shield Tunneling Circulation System Efficiency on the Influence of Mud. Tunnel Construction, 2013, 33(11): 928-932.

[23] Shunhua Zhou, Xue Li, Chang Ji, Junhua Xiao. Back-fill Grout Experimental Test for Discharged Soils Reuse of the Large-diameter Size Slurry Shield Tunnel. KSCE Journal of Civil Engineering, 2017, 21(3):725-733.

[24] R. Di Felice. The Voidage Function for Fluid-particle Interaction Systems. International Journal of Multiphase Flow, 1994, 20(1):153-159.

[25] B. P. B Hoomans, J. A. M Kuipers, W. J Briels, W. P. M.van Swaaij. Discrete particle simulation of bubble and slug formation in a two-dimensional gas-fluidised bed: A hard-sphere approach. Chemical Engineering Science, 1996, 51(1):99-118.

[26] Nan Gui, Xingtuan Yang, Jiyuan Tu, Shengyao Jiang. An extension of hard-particle model for three-dimensional non-spherical particles: Mathematical formulation and validation. Applied Mathematical Modelling, 2016, 40(4):2485-2499.

[27] Hongyan Li. Research and application of discrete element dry-wet particle model. Beijing, China Agricultural University, 2004.

[28] K. L. Johnson, , K. Kendal, A. D. Roberts. Surface energy and the contact of elastic solids. Proc. R. Soc. Lond. 1971,324 (1558): $301-313$

[29] Jiapeng Tang. ANSYS FLUENT 16.0 Super Learning Manual. Beijing, 2016.

[30] DEM Solutions Ltd. EDEM 2017 User Guide. Edinburgh, 2016.

[31] Zhou Lu, Xuedong Liu, Pan Bing. Numerical Simulation of Cylindrical Particles Conveying in Curved Ducts Using CFD-DEM Coupled Approach. China Powder Science and Technology, 2011, 10: 65-69. 